\title{
Wakefield measurement using principal component analysis on bunch-by-bunch information during transient state of injection in a storage ring
}

\author{
Zhichu Chen, ${ }^{1,2, *}$ Yong Yang, ${ }^{1}$ Yongbin Leng, ${ }^{1,2, \dagger}$ and Renxian Yuan ${ }^{1,2}$ \\ ${ }^{1}$ Shanghai Institute of Applied Physics, Chinese Academy of Sciences, Shanghai 201800, China \\ ${ }^{2}$ Shanghai Synchrotron Radiation Facility, Chinese Academy of Sciences, Shanghai 201204, China
}

(Received 29 August 2014; published 14 November 2014)

\begin{abstract}
Wakefields and beam instabilities are important issues for a storage ring and are described by various theoretical formalisms. Direct measurements of the beam motion related to different dynamical mechanisms are a useful input to accelerator optimization. This paper reports on an experimental method based on a simplified wakefield model, where bunch-by-bunch position information were monitored during the transient injection process at the Shanghai Synchrotron Radiation Facility. Processing the bunch-by-bunch data by means of the principal component analysis allowed for immediate operational improvements-such as in situ compensation of the kicker leakage, and energy matching between the booster and the storage ring.
\end{abstract}

DOI: 10.1103/PhysRevSTAB.17.112803

PACS numbers: 29.20.db, 29.27.Bd, 29.85.Fj

\section{INTRODUCTION}

Interactions of beams with its surroundings are critical to beam stabilities, especially for multibunch storage rings. Wakefields in regular structures have analytic solutions and various models have been presented in the past decades to study the wakes and impedances theoretically [1-7], but the calculation of the detailed wakefields are tedious and time consuming by simulations in finite element analysis softwares such as MAFIA or CST. Trapped modes of the wakefield are difficult to measure by wire based bench measurement $[8,9]$. Although beam based measurements do not have this shortcoming, crafty designs, like the techniques used in ASSET [10], are necessary.

The injection system of a storage ring usually consists of a set of beam kickers. It changes the momentum of a bunch to meet the injected one, and then changes it back to the original orbit with the injected bunch. Unfortunately, the kickers of some injection systems, such as the ones at the Shanghai Synchrotron Radiation Facility (SSRF) are not perfectly mounted, aligned or configured, thus there will be residual betatron oscillations.

On the other hand, the residual oscillations could be regarded as excitations and the wakefields would affect all the bunches including itself. The betatron oscillations are evanescent in a storage ring, especially when there is a transverse feedback system. The signal-to-noise ratio might become acceptable in the injection transient process.

\footnotetext{
*chenzhichu@sinap.ac.cn

†lengyongbin@sinap.ac.cn
}

Published by the American Physical Society under the terms of the Creative Commons Attribution 3.0 License. Further distribution of this work must maintain attribution to the author(s) and the published article's title, journal citation, and DOI.
The wake oscillation could always be expanded as power series of the displacement of the drive bunch. The brief explanation of a simplified linear wake model is given in Sec. II where the higher order terms of the power expansion are ignored. If there is an electronics that can fetch the bunch-by-bunch positions of the beam during the injection, the wakefield along the storage ring might be measured and instabilities introduced by the flaw of the kickers could hopefully be adjusted.

\section{WAKEFIELD MODELING}

The transverse motion of the charged particles in a storage ring is periodically restrained by the damping forces and the quadrupole fields, so the equation of motion has the form of

$$
x^{\prime \prime}+p(s) x^{\prime}+q(s) x=0,
$$

where $s$ is the longitudinal coordinate, $x$ the abscissa or the ordinate, $x^{\prime}=\frac{\mathrm{d} x}{\mathrm{~d} s}=\frac{\mathrm{d} x}{\mathrm{~d} t} / \frac{\mathrm{d} s}{\mathrm{~d} t}$ the generalized transverse velocity and $p(s+L)=p(s), q(s+L)=q(s)$ both $L$-periodic functions determined by the lattice and the structure of the storage ring. The space charge effect and the image charge/ current contribution to the beam motion [11] is ignored here as higher order terms.

Define

$$
X_{0}=\left(\begin{array}{c}
x_{0} \\
x_{0}^{\prime}
\end{array}\right)
$$

and Eq. (1) can be written as the following homogeneous linear system:

$$
X_{0}^{\prime}=A(s) X_{0}
$$

where 


$$
A(s)=\left(\begin{array}{cc}
0 & 1 \\
-q(s) & -p(s)
\end{array}\right) .
$$

The Floquet theory [12], p. 189, and the Liouville's formula [12], p. 152, show that there will be a quasiperiodic fundamental matrix solution,

$$
\Phi_{0}(s)=R(s) e^{s B},
$$

where $R(s)$ is an $L$-periodic matrix function and

$$
\operatorname{tr} B=\frac{1}{L} \int_{0}^{L} \operatorname{tr} A(s) \mathrm{d} s=-\frac{1}{L} \int_{0}^{L} p(s) \mathrm{d} s .
$$

Since $B$ is similar to its Jordan canonical form $J=\lambda I+N$ where $I$ is the $2 \times 2$ identity matrix and $N$ a traceless nilpotent matrix, $\operatorname{tr} B=\operatorname{tr} J=2 \lambda$. The damping forces require that $p(s) \geq 0$ so that $\lambda \leq 0$. It can be easily seen that the solution is a linear combination of the columns of the principal fundamental matrix:

$$
\Psi_{0}(s)=e^{\lambda s}\left(\begin{array}{cc}
r(s) & 0 \\
r^{\prime}(s) & r(s)
\end{array}\right)\left(\begin{array}{cc}
\cos (k s) & \frac{\sin (k s)}{k} \\
-k \sin (k s) & \cos (k s)
\end{array}\right),
$$

where the $L$-periodic function $r(s)$ is normalized so that $r(0)=1$ and $r^{\prime}(0)=0$. The beam position measured each turn at a fixed location is the upper element of $\Psi_{0}\left(s_{0}+n L\right) v$, where $v$ is a constant vector determined by the initial condition, and could be regarded as a sampling from a continuously damped sinusoid (as shown in Fig. 1):

$$
x_{0}^{n}\left(s_{0}\right)=x\left(s_{0}+n L\right)=C_{0} r\left(s_{0}\right) \cos \left(n k L+\theta_{0}^{0}\right) e^{n L \lambda} .
$$

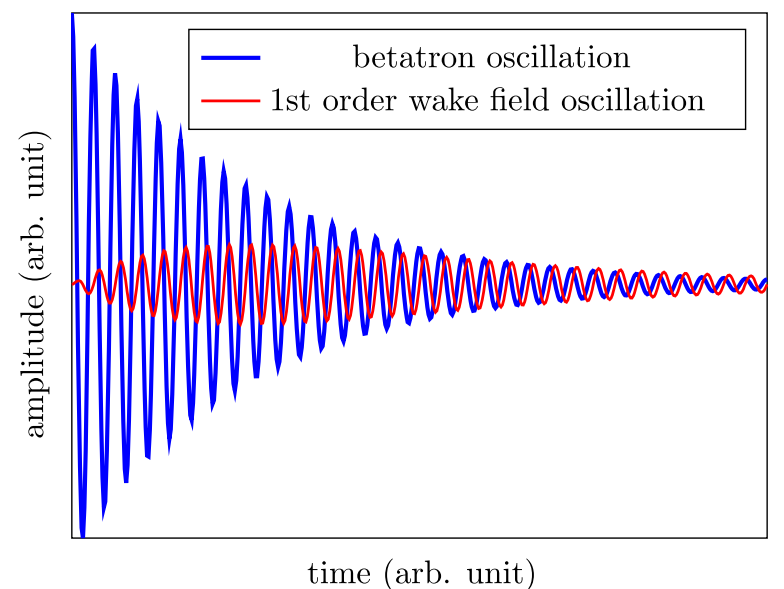

FIG. 1. Illustration of the betatron damping oscillation and the first order wakefield motion.

To describe the motion of the successive bunch affected by the free bunch, let us consider the linear approximation of the wakefield and the equation of the motion could be written as

$$
X_{1}^{\prime} \simeq A(s) X_{1}+a_{1,0} Y_{0},
$$

where

$$
X_{1}=\left(\begin{array}{c}
x_{1} \\
x_{1}^{\prime}
\end{array}\right), \quad Y_{0}=\left(\begin{array}{c}
0 \\
x_{0}
\end{array}\right)=\left(\begin{array}{ll}
0 & 0 \\
1 & 0
\end{array}\right) \Psi_{0} v,
$$

and $a_{1,0}$ a constant coefficient which denotes the wakefield force propagated from the previous bunch. Using the variation of parameters formula [12], p. 179, the solution of the inhomogeneous system could be written as

$$
\begin{aligned}
\phi_{1}(s) & \simeq \Psi_{0}(s) \Psi_{0}^{-1}(0) \Psi_{0}(0) v^{1}+\Psi_{0}(s) \int_{0}^{s} \Psi_{0}^{-1}(t) a_{1,0} Y_{0}(t) \mathrm{d} t \\
& =\Psi_{0}(s) v^{1}+a_{1,0} \Psi_{0}(s)\left(\begin{array}{cc}
-\frac{\sin ^{2}(k s)}{2 k^{2}} & \frac{\sin (k s) \cos (k s)-k s}{2 k^{3}} \\
\frac{\sin (k s) \cos (k s)+k s}{2 k} & \frac{\sin ^{2}(k s)}{2 k^{2}}
\end{array}\right) v^{0} \\
& =\Psi_{0}(s) v^{1}+a_{1,0} \frac{e^{\lambda s}}{2 k^{2}}\left(\begin{array}{cc}
r(s) & 0 \\
r^{\prime}(s) & r(s)
\end{array}\right)\left(\begin{array}{cc}
k s \sin (k s) & \frac{\sin (k s)-k s \cos (k s)}{k} \\
k[\sin (k s)+k s \cos (k s)] & k s \sin (k s)
\end{array}\right) v^{0}
\end{aligned}
$$

and thus the beam position at $s_{0}$ could be written as (as shown in Fig. 1)

$$
x_{1}^{n}\left(s_{0}\right)=x_{1}\left(s_{0}+n L\right) \simeq\left[C_{1,0} \cos \left(n k L+\theta_{1}^{0}\right)+C_{1,1} n \cos \left(n k L+\theta_{1}^{1}\right)\right] \times r\left(s_{0}\right) e^{n L \lambda} .
$$

It can be easily derived that the beam position of the $(i+1)$ th bunch affected by the previous $i$ bunches which are also affected by their preceding bunches at a fixed location is of the form

$$
x_{i}^{n}\left(s_{0}\right) \simeq r\left(s_{0}\right) e^{n L \lambda} \sum_{j=0}^{i} C_{i, j} n^{j} \cos \left(n k L+\theta_{i}^{j}\right) .
$$



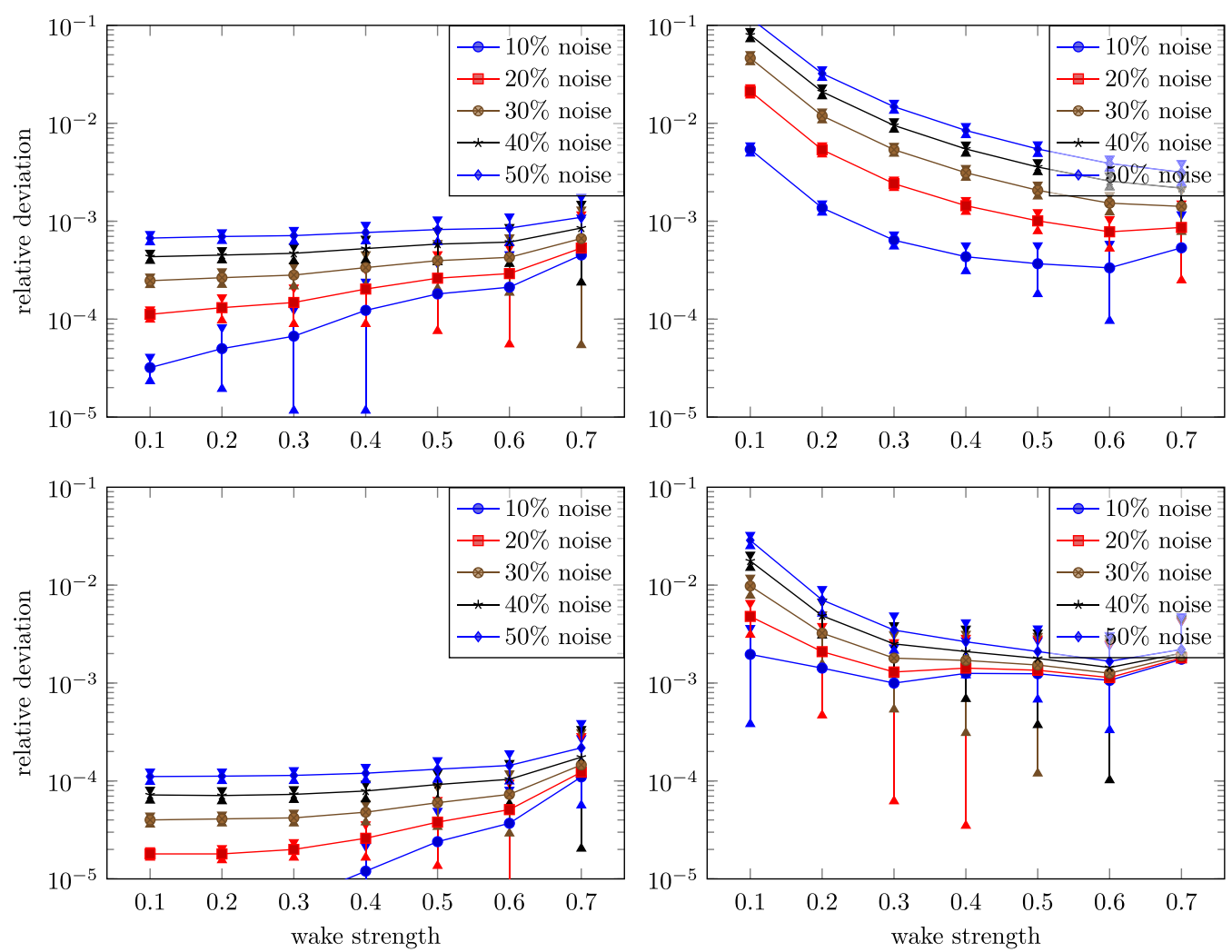

FIG. 2. Relative differences between the temporal vectors or the spatial vectors of the extracted betatron mode or the wake mode and the original ones. Upper left: $\left(u_{1}-K\right) / K$; upper right: $\left|\left(v_{1}-A\right) / A\right|$; lower left: $\left|\left(u_{2}-L\right) / L\right|$; lower right: $\left(v_{2}-B\right) / B$. The abscissa is $b / a$, meaning the ratio of the amplitude of the wake oscillation to the amplitude of the betatron oscillation. Different lines had different noise levels $c / a$ contributed to the simulation.

It could be approximately expressed as the sum of the betatron damping oscillation and the first order wakefield oscillation, which is invoked by its previous bunches just like Eq. (12), after omitting the higher order terms.

Generally, principal component analysis of the turn-byturn beam position monitor (BPM) data will extract two betatron oscillation modes, due to the phase advances between BPMs. But the evanescent betatron oscillation has already been damped and it is not detectable before the injection. The initial condition is not arbitrary in that case. The bunches are kicked at $s=0$ so the betatron oscillations are all cosinelike and share the same oscillation phase, which means that the linear combination coefficient can be written as $v^{i}=\left(v_{1}^{i}, 0\right)^{T}$. So the betatron mode extracted from the bunch-by-bunch data will only have 1 degree of freedom. Comparing solutions (7) and (11), we can see that although the first-order wakefield oscillation $\left[\propto s e^{\lambda s} \sin (k s)\right]$ depends on the betatron oscillation $\left[\propto e^{\lambda s} \cos (k s)\right]$, they are linearly unrelated. Inspired by model-independent analysis (MIA) [13,14], we found that these modes could be separated by using the singular value decomposition (SVD). Once the motions are separated, the spatial vector [13] of the betatron oscillation could be used to describe the variation of the magnetic fields of the kickers of the injection system.
To verify that the two modes are separable in discrete data, a computer simulation had been made before the experiments to estimate the performance of the decomposition. Damped betatron oscillations, mixed with wake oscillations and random noise, were used to simulate the behavior of the bunches. Different amplitudes of the betatron oscillation of all bunches were chosen during the simulation. Since the range of the wakefield and the charges of the bunches were unknown, random distribution along bunches had been used. The signals had the following form:

$$
x_{i}(n)=A_{i} e^{-n / N} \cos (n \vartheta)+B_{i} n e^{-n / N} \sin (n \vartheta)+C_{i} R(n),
$$

where $A_{i}, B_{i}$ and $C_{i}$ were random scalars and $R(n)$ random noise. In this simulation, $A_{i}$ 's had a unit normal distribution $N(0,1) .{ }^{1} B_{i}$ 's and $C_{i}$ 's also had normal distributions $N\left(0, \sigma_{b}\right)$ and $N\left(0, \sigma_{c}\right)$. Signals using different $\sigma_{b}$ 's and $\sigma_{c}$ 's were generated before they were decomposed into principal components:

\footnotetext{
${ }^{1} N(\mu, \sigma)$ is the normal distribution where $\mu$ and $\sigma$ are the mean and the standard deviation of the distribution.
} 


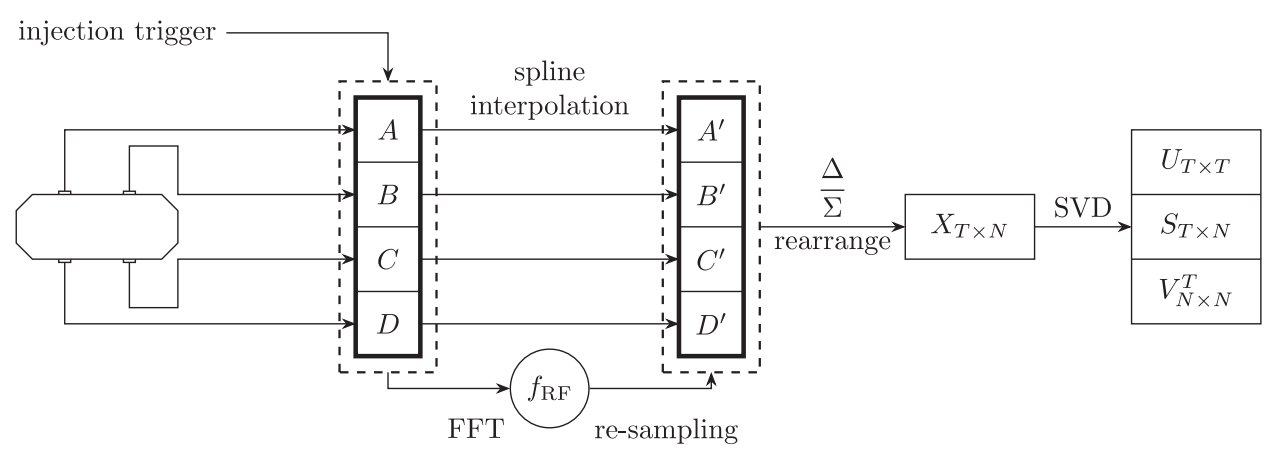

BPM

Oscilloscope

FIG. 3. Measurement scheme and data preparing. The oscilloscope is attached to the electrodes of the BPM and it fetches the original waveforms over 10000 turns right after the injection. After resampling the spline interpolation of the original waveform of each button at the frequency of $f_{\text {rf }}$, the positions of each bunch is calculated and arranged for further analysis.

$$
\begin{aligned}
& a K \times A+b L \times B+c R \times C \\
& \rightarrow U S V^{T} \\
& =\left(\begin{array}{llll}
u_{1} & u_{2} & u_{3} & \cdots
\end{array}\right)\left(\begin{array}{cccc}
a & 0 & 0 & \cdots \\
0 & b & 0 & \ldots \\
0 & 0 & c & \ldots \\
\vdots & \vdots & \vdots & \ddots
\end{array}\right)\left(\begin{array}{c}
v_{1}^{T} \\
v_{2}^{T} \\
v_{3}^{T} \\
\vdots
\end{array}\right),
\end{aligned}
$$

where $K$ is the unitary column vector of the damped betatron oscillation; $L$ is the unitary column vector of the wake oscillation; $R$ is the unitary column vector of the noise; $A, B$ and $C$ are the unitary row vectors of $A_{i}, B_{i}$ and $C_{i}$, respectively; and $U, S$ and $V$ are the temporal vectors [13], singular matrix and spatial vectors, respectively.

The decomposed betatron and wake oscillations were compared with the theoretical ones (as shown in Fig. 2). The results showed that the relative differences between the extracted temporal vectors and the original signals were no greater than $10^{-3}$ in the simulation. The differences between the spatial vectors and the original distributions were less than $10^{-1}$. The performance of the decomposition relied on the ratios of wake and noise to betatron oscillation.

\section{MEASUREMENT APPARATUS}

The storage ring at the SSRF consists of 20 cells. The beam is injected at cell 1 and the bunch-by-bunch position measurement system is located at cell 10. The bunch positions at these locations are related by a constant matrix so that the kickers' field could be constructed in situ by using the spatial vector of the pure betatron oscillation mode at the measurement location.

Since there were few standard electronics that could measure the beam positions bunch by bunch with both high sampling rate and huge memory capacity to store the data, an oscilloscope was chosen to record the signals from the four buttons of a BPM and to output the corresponding bunch positions, as shown in Fig. 3. The rf frequency obtained from the discrete Fourier transformation of the raw waveform was used to determine the bunch interval. The sampling rate of the oscilloscope was not an integer multiple of the rf frequency $(499.654 \mathrm{MHz}$ in our storage ring) so cubic spline interpolation was used to obtain the bunch positions at accurate signal phases. The bunch-bybunch position series was rearranged and transformed into turn-by-turn position information of each bunch so that the bunch position matrix was ready for further beam dynamics analysis [15].

\section{EXPERIMENTS AND RESULTS}

The experiment does not need special configurations of the machine, so the data were collected unnoticed during daily top-off operations. Bunches were injected every 10 minutes and the data acquisition was triggered slightly before the injection. The typical results plotted in this section were processed by using the data recorded during the injection at 14:50 on July 15, 2014 unless otherwise noted.

After the bunch position matrix $X$ (consists of horizontal motion waveforms of all bunches, typically shown in Fig. 4) described in the previous section is constructed, it is decomposed into three matrices $X \rightarrow U S V^{T}$ (as shown in Fig. 3), where $U$ and $V$ are unitary matrices and $S$ is diagonal. Similar to MIA, the column vectors in $U$ are called the temporal vectors and the column vectors in $V$ are called the spatial vectors.

Based on the singular values (see Fig. 5), only four major modes (as shown in Figs. 6 and 7) with relatively large singular values were worth studying in this experiment.

The envelope of the first mode is an exponential decay, so it could be considered as a clean betatron damping oscillation invoked exclusively by the kickers according to Eq. (13). The spatial vector indicated that the magnetic fields influencing the bunches were not constant. In this experiment, the 417 th bunch is not affected by the kickers 


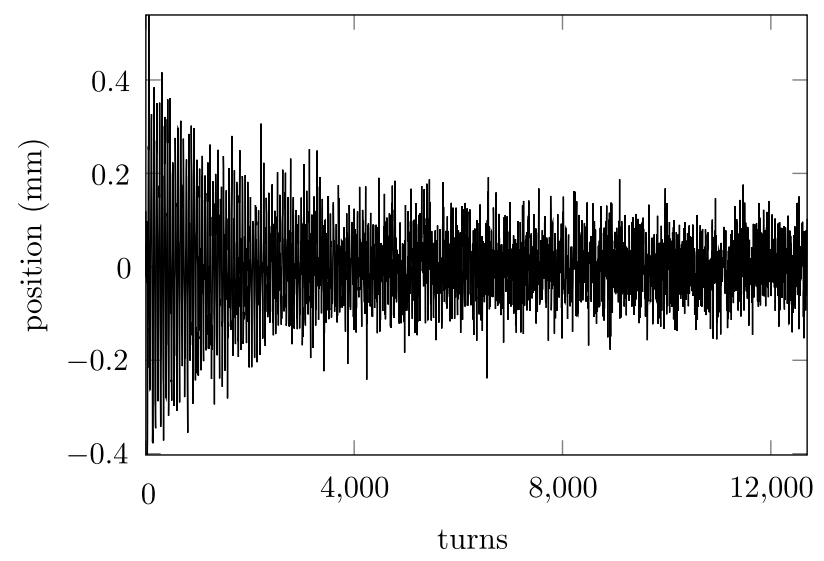

FIG. 4. Typical waveform of some random bunch at the measurement spot.

at all. The kickers at the SSRF is optimized so that the bunch to be injected (the 141st bunch in this experiment) would always be at the maximum phase and the matching between the fact and the spatial vector confirmed that SVD could extract the betatron oscillation mode from the bunch-by-bunch data.

Repeating this experiment, we have found that although the kickers' field has an irregular temporal distribution, it is stable with respect to the injected bunch. The field could be calibrated by using multiple spatial vectors during different injections with the knowledge of the positions of the injected bunches. As an illustration, three sequential measurements were used as snapshots of the full field of 720 buckets, as shown in Fig. 8. Some spatial vectors had been flipped upside down to coincide with each other and the flipping is harmless because it would only change the sign or phase of the corresponding temporal vector.

The envelope of the second mode is a multiplication of the turn number and an exponential decay, thus it is caused by the first order wakefield according to Eq. (13). The amplitude of this mode is about a half of the betatron

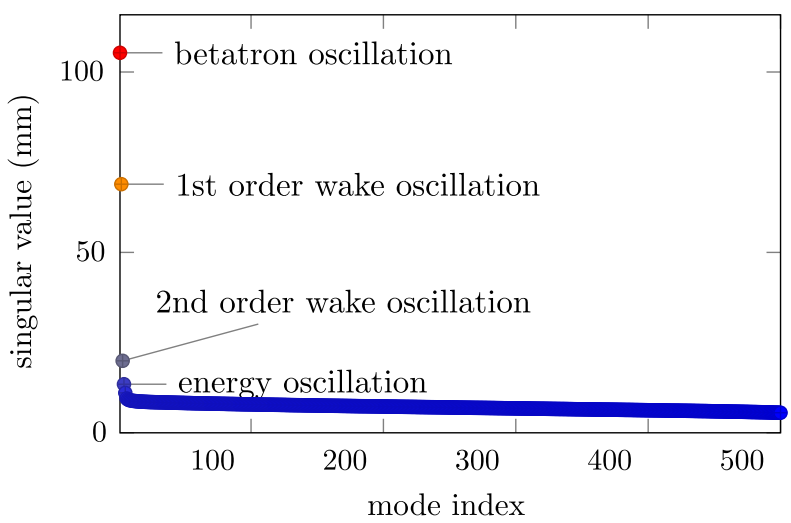

FIG. 5. Singular values of the separated modes. The noise floor is about $7.3 \mathrm{~mm}$ and the signal-to-noise ratio of the betatron oscillation is about $11.6 \mathrm{~dB}$.

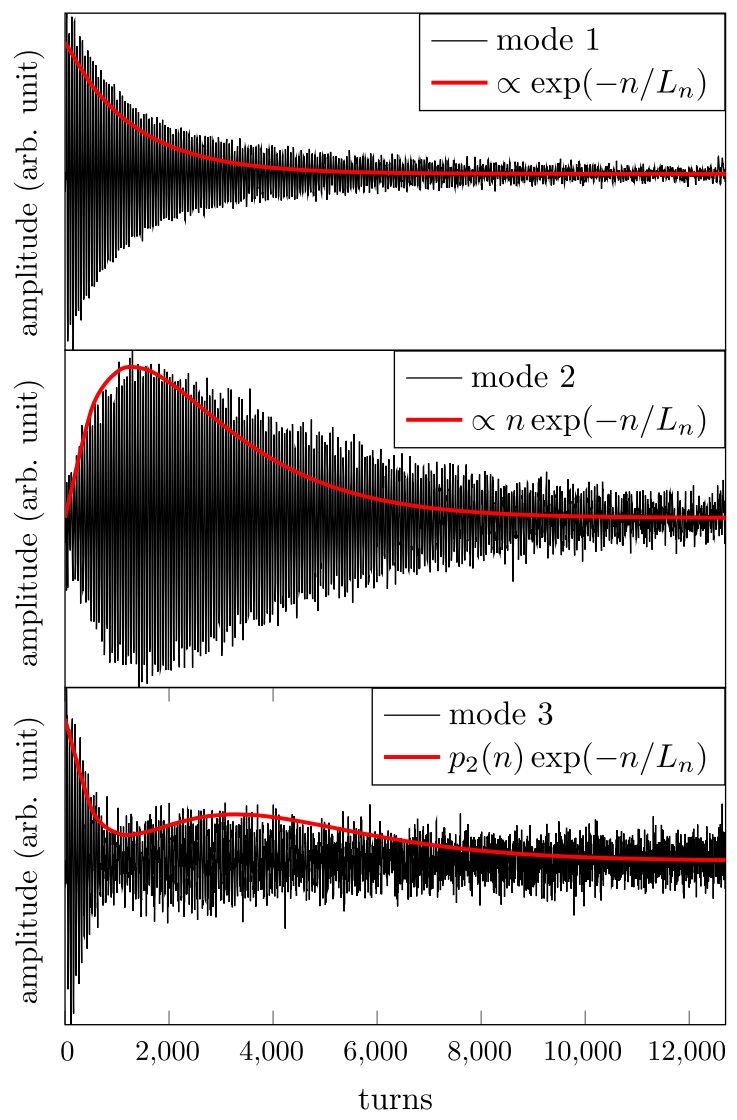

FIG. 6. The first three principal components of the oscillation after the injection. The red auxiliary lines illustrate that the envelopes of the temporal vectors satisfy the general solution (13). $L_{n}$ is a constant and $p_{2}(n)$ is a quadratic polynomial of the turn number $n$.

oscillation and the phase difference between them showed that the total wakefield seen by a bunch has a long range. The dynamics of the producing and the propagating of the wakefield could be precisely studied by measuring this mode under different filling patterns.

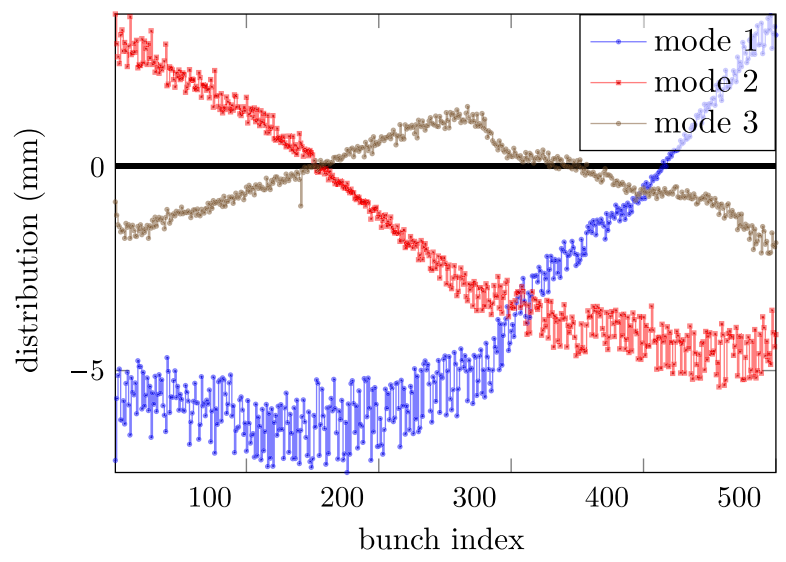

FIG. 7. Distributions of the first three modes along the bunches. 


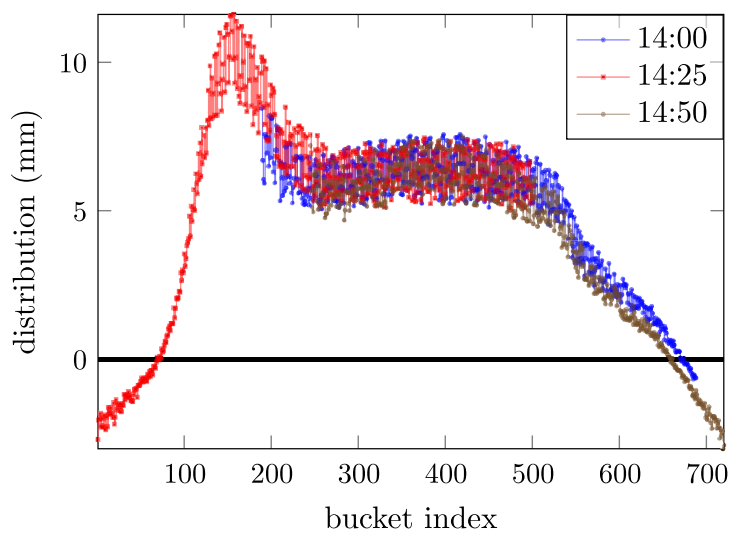

FIG. 8. Kicker field reconstruction based on three sets of data acquired at 14:00, 14:25 and 14:50, respectively, on July 15, 2014.

The wake oscillation of a bunch is determined by the betatron oscillations of all bunches. The propagation coefficient of the wakefield is considered a constant once the drive bunch and the witness bunch are decided, so the amplitude of second mode of a bunch is a linear combination of the amplitudes of the first modes of all bunches (including itself):

$$
S_{2}^{2} V_{i}^{2}=\sum_{k=1}^{N} \alpha_{i}^{k} S_{1}^{1} V_{k}^{1},
$$

where $S_{2}^{2}$ is the singular value of the wake mode (the second principal component in these experiments), $V_{i}^{2}$ is the $i$ th element of the spatial vector of the wake mode, $\alpha_{i}^{k}$ is the propagation coefficient from the betatron oscillation of the $k$ th bunch to the wake oscillation of the $i$ th bunch, $S_{1}^{1}$ is the singular value of the betatron mode (the first principal component in these experiments), $V_{k}^{1}$ is the $k$ th element of the spatial vector of the betatron mode, and $N$ is the bucket number of the storage ring. The $\alpha$ matrix can be obtained by solving the linear regression problem in Eq. (16).

The envelope of the third component is a multiplication of a quadratic polynomial of the turn number and an exponential decay, and it is a higher order mode caused by the second component according to Eq. (13). Higher order modes where the degree of the polynomial is greater than 2 had not been observed in the experiment or, more likely, were mixed in the first three principal components.

The fourth component is coupled from the energy oscillation due to dispersion based on the waveform (as shown in Fig. 9) and its spectrum. The spatial vector showed that the injected bunch had a very large energy oscillation component. Combined with the filling pattern information, the injected charge is proportional to the energy spread of the bunch (as shown in Fig. 10) [16], which implied that the component is due to the energy mismatch between the storage ring and the booster.
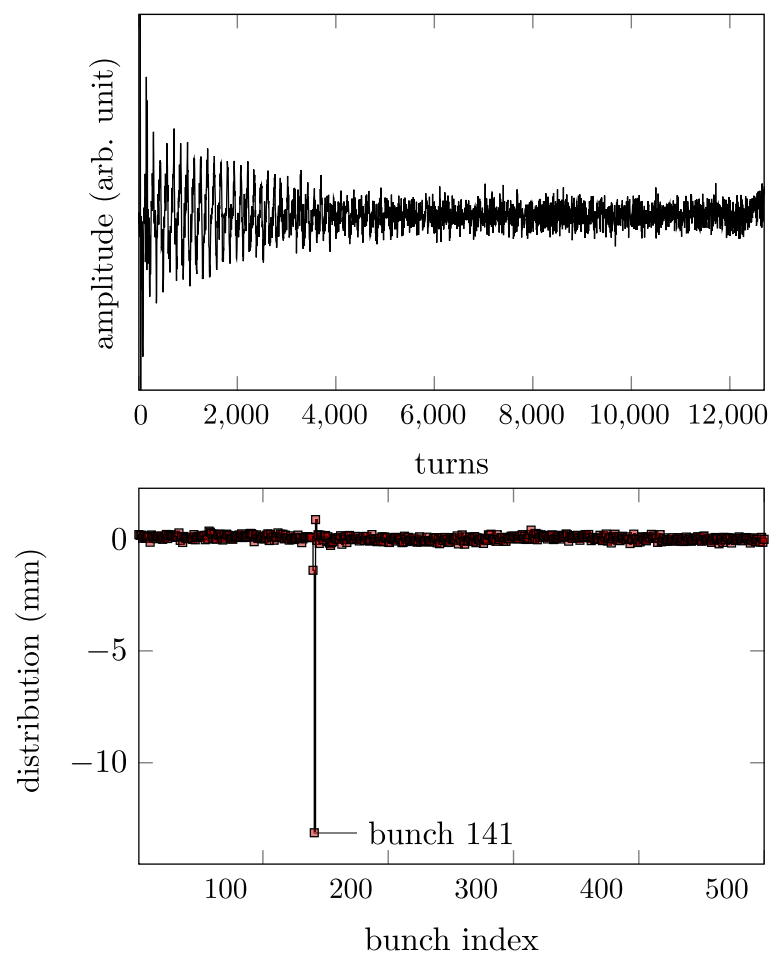

FIG. 9. Temporal vector (top) and spatial vector (bottom) of the energy oscillation extracted from the original data.

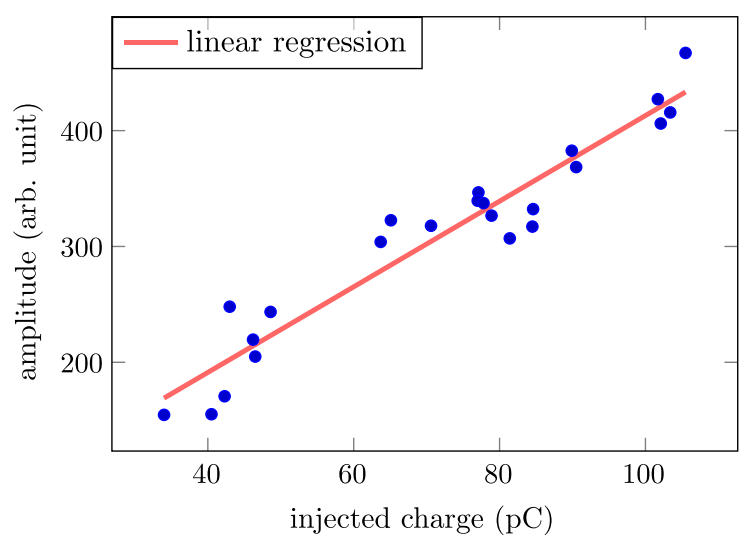

FIG. 10. Linear relation between the amplitude of the energy oscillation extracted from the original data and the charge of the injected bunch.

\section{CONCLUSION}

The transverse motions of the beam after the injection consist of many physical oscillation modes and unwanted noise. This paper proposed an experimental method to separate these modes.

A series of experiments had roughly confirmed that the linear dipole wakefield model stated in Sec. II is adequate in analyzing multibunch motions in a storage ring during injection without special setups and additional machinestudy hours. Those higher order modes were separated and 
observed from the raw position data by using SVD inspired by MIA without disturbing the ordinary operation of a light source.

To apply this method, a specialized digital beam position monitor (DBPM) is under development. The DBPM would separate the physical modes in its field programmable gate array module after acquiring the positions of the bunches after each injection. Information of the betatron modes would be used to compensate the errors of the kickers. Information of the energy oscillation modes would be used to fine-tune the energy of the booster. After accumulating enough samples of the betatron modes and the wake modes, the interaction strength between bunches will be studied.

\section{ACKNOWLEDGMENTS}

This work was supported by the National Natural Science Foundation of China (No. 11375255).

[1] S. A. Heifets and S. A. Kheifets, Rev. Mod. Phys. 63, 631 (1991).

[2] B. W. Zotter and S. A. Kheifets, Impedances and Wakes in High-Energy Particle Accelerators (World Scientific, Singapore, 1998).

[3] I. Zagorodnov and T. Weiland, Phys. Rev. ST Accel. Beams 8, 042001 (2005).

[4] G. Stupakov, K. L. F. Bane, and I. Zagorodnov, Phys. Rev. ST Accel. Beams 10, 054401 (2007).

[5] K. L. F. Bane, G. Stupakov, and I. Zagorodnov, Phys. Rev. ST Accel. Beams 10, 074401 (2007).
[6] G. V. Stupakov and I. A Kotelnikov, Phys. Rev. ST Accel. Beams 12, 104401 (2009).

[7] G. Stupakov, K. L. F. Bane, and I. Zagorodnov, Phys. Rev. ST Accel. Beams 14, 014402 (2011).

[8] F. Caspers, in Frontiers of Particle Beams: Intensity Limitations, edited by M. Dienes, M. Month, and S. Turner (Springer, Berlin, 1992), Vol. 400.

[9] R. M. Jones, Phys. Rev. ST Accel. Beams 12, 104801 (2009).

[10] C. Adolphsen, K. Bane, T. Higo, K. Kubo, R. Miller, R. Ruth, K. Thompson, and J. Wang, Phys. Rev. Lett. 74, 2475 (1995).

[11] A. W. Chao, Physics of Collective Beam Instabilities in High Energy Accelerators, 1st ed., edited by M. Month, Wiley Series in Beam Physics and Accelerator Technology (John Wiley \& Sons, Inc., New York, 1993).

[12] C. Chicone, Ordinary Differential Equations with Applications, 2nd ed., edited by J. E. Marsden, L. Sirovich, and S. S. Antman, Texts in Applied Mathematics (Springer, New York, 2006), Vol. 34.

[13] J. Irwin, C. x. Wang, Y. T. Yan, K. L. F. Bane, Y. Cai, F. J. Decker, M. G. Minty, G. V. Stupakov, and F. Zimmermann, Phys. Rev. Lett. 82, 1684 (1999).

[14] C. x. Wang, Ph.D. dissertation, Stanford University, 1999.

[15] Y. Yang, Y. Leng, Y. Yan, and N. Zhang, in Proceedings of the 4th International Particle Accelerator Conference, edited by Z. Dai, C. Petit-Jean-Genaz, V. R. W. Schaa, and C. Zhang (JACoW, Shanghai, China, 2013), pp. 595-597.

[16] Y. Leng, Y. Yang, N. Zhang, Y. Yan, and R. Yuan, in Proceedings of the 2nd International Beam Instrumentation Conference, edited by I. Costa, M. Marx, L. Liljeby, R. Müller, I. Martin, and S. Weizenkamp (Diamond Light Source, Oxford, UK, 2013), pp. 746-748. 